\title{
SPATIAL CORRELATION FUNCTIONS OF BRIGHT RADIO GALAXIES
}

You--yuan Zhou and Yi-peng Jing

Center for Astrophysics

University of Science and Technology of China

Hefel. Anhui, China

Because of their large separation bright radio galaxies characterize the structure of the universe on the scales of clusters and superclusters. In order to calculate the spatial correlation function we choose radio galaxies in radio surveys. for which the redshift values have been measured. One is from Bright Radio Sources at $178 \mathrm{MHz}$ (Llang. Rilley and Longair 1983), and another is from All-Sky Catalogue of Bright Extragalactic Radio Sources at 2.7 GHz (Wall and Peacock 1985).

For the samples with $z<0.1, z<0.3$ and $z<0.5$ the two-point spatial correlation functions have the following features.

1. In $r<30 \mathrm{~h}^{-1} \mathrm{Mpc}$ there is comparatively large correlation. which is similar to that of Abell clusters, especially for the $2.7 \mathrm{GHz}$ samples (see fig. 1). This shows that a large number of bright radio galaxies are located in rich clusters (Seldner \& Peebles 1978).

2. From $100 \mathrm{~h}^{-1} \mathrm{Mpc}$ to $170 \mathrm{~h}^{-1} \mathrm{Mpc}$ the correlation functions obviously increase, and at maximum points exceed $1 \sigma$ in comparison to Monte--Carlo sampling. This shows that radio galaxies are located in superclusters.

From the correlation functions of samples with $0.1 \leqslant z \leqslant 0.3$ and $0.2 \leqslant z \leqslant 0.5$. We find that there is no obvious correlation above supercluster size up to $500 \mathrm{~h}^{-1} \mathrm{Mpc}$. It shows that the universe above the supercluster size is structureless. The conclusion was also obtained from the space distribution of QSOs (Zhou. Fang. Deng. and $X_{\theta}$ ).

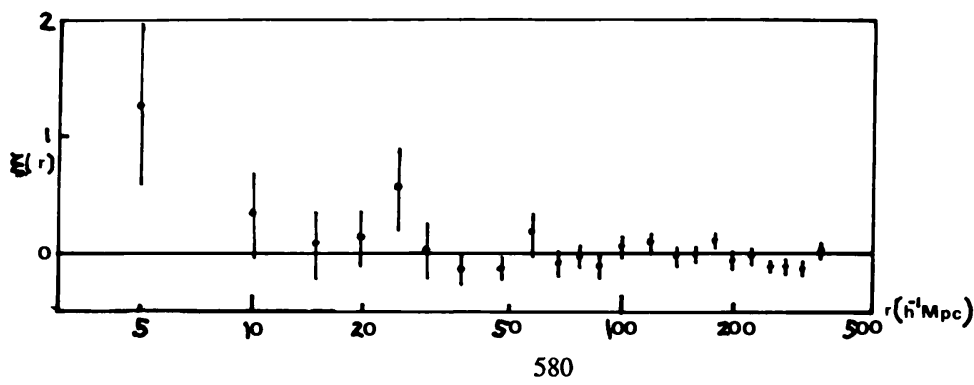

J. Audouze et al. (eds.), Large Scale Structures of the Universe, 580.

(c) 1988 by the IAU. 